\title{
A relação entre sucesso de novos produtos, orientação para o mercado e performance empresarial
}

\author{
Cláudio Hoffmann Sampaio \\ Mestrado em Administração e Negócios - PUC/RS \\ csampaio@pucrs.br
}

Marcelo Gattermann Perin

Mestrado em Administração e Negócios - PUC/RS

$\underline{\text { mperin@pucrs.br }}$

Gabriela Cardozo Ferreira

Mestrado em Administração e Negócios - PUC/RS

gcferreira@pucrs.br

\begin{abstract}
Apesar da consolidação teórica do tema orientação para mercado e sua influência no desempenho organizacional, uma série de estudos vem demonstrando que há limites na sua capacidade para impactar diretamente algumas facetas da performance organizacional, sugerindo a existência de variáveis mediadoras nesta relação, entre elas a inovação. Nesta linha, este artigo objetivou avaliar a relação da orientação para mercado com a performance empresarial, sob a mediação do nível de sucesso de novos produtos. Para tanto, um modelo teórico desenvolvido foi testado pela aplicação da técnica de modelagem de equações estruturais, em um estudo que envolveu empresas de diversos setores econômicos do Brasil. Os resultados apontam para um impacto indireto da orientação para mercado sobre a participação de mercado, mediada pelo sucesso de novos produtos. Também foi registrado o impacto indireto do sucesso de novos produtos sobre a lucratividade, tendo como variável mediadora a participação de mercado.
\end{abstract}

Palavras-chave: sucesso de novos produtos; orientação para mercado; performance organizacional

In spite of the theoretical consolidation of market orientation and its influence on organizational performance, a series of studies demonstrates that there are limits in its capacity for representing direct impact in some performance dimensions. This suggests the mediation of other variables such as innovation. This article aims at evaluating the relationship between market orientation and the main enterprises' performance dimensions, under the mediation of innovation success level. A theoretical model was tested using structural equations modeling involving Brazilian companies from several economic sectors. Results point out a direct impact of market orientation on companies ' profitability, as well as an indirect impact on its market share, both mediated by new products success. It was also registered an indirect impact of new products success on profitability, with market share as a mediating variable.

Key-words: new product success; market orientation; performance

\section{Introdução}

Buscar entender os antecedentes do sucesso de novos produtos e da performance empresarial tem sido um desafio aos pesquisadores de administração (BARCZAK; KAHN; MOSS, 2006; HARMANCIOGLU; McNALLY; CALANTONE; DURMUSOGLU, 2007; PALADINO, 2007; PATTIKAWA; VERWAAL; COMMANDEUR, 2006; URDAN; OSAKU, 2005). Entre esses antecedentes, o construto de orientação para o mercado aparece como um de forte poder explicativo (BAKER; SINKULA, 2005; HOMBURG; PFLESSER, 2000; HOOLEY; GREENLEY; CADOGAN; FAHY, 2005). Como decorrência, a orientação para mercado recebeu substancial interesse acadêmico e de profissionais de administração estratégica ao longo dos últimos anos 
(BAKER; SINKULA, 2007), especialmente na sua relação com o construto de performance organizacional (LANGERAK, 2003; UNCLES, 2000), tendo gerado um número significativo de trabalhos e um claro avanço na teoria sobre o tema (BAKER; SINKULA, 2005; BAKER; SINKULA, 2007; SLATER; NARVER, 2000).

A verificação empírica da relação entre orientação para o mercado e performance tem sido foco central de diversos estudos, conduzidos em diferentes indústrias, setores e contextos culturais. Inicialmente, a maioria dos estudos que abordava essa relação foi realizada nos EUA. Entretanto, o argumento de que trabalhos realizados em ambientes diferentes poderiam apresentar resultados distintos serviu de motivação para que pesquisas similares, que verificassem a relação entre orientação para o mercado e performance, fossem realizadas em outros países, inclusive no Brasil, em especial nos estudos realizados por Perin e Sampaio (por exemplo, PERIN; SAMPAIO, 2001; PERIN; SAMPAIO, 2003; PERIN; SAMPAIO; FALEIRO, 2002). De uma maneira geral, observa-se uma relação positiva entre orientação para o mercado e performance empresarial.

$\mathrm{Na}$ maioria das pesquisas relatadas, contudo, a orientação para o mercado tem sido relacionada a medidas de performance, uma de cada vez (LANGERAK, 2003). Estudos de redes nomológicas, incluindo orientação para o mercado e múltiplas medidas de performance, relacionando variáveis mediadoras, são raros (BAKER; SINKULA, 2005).

Entre os requisitos que aparecem como fundamentais para que as empresas obtenham sucesso competitivo em seus mercados aparece a necessidade de reagir com sucesso às demandas, além de influenciar o ambiente no qual elas estão inseridas (SLATER; NARVER, 1995). A crença nessa afirmação tem, há muito tempo, emprestado relevância ao construto de inovação e de sucesso de novos produtos (HURLEY; HULT, 1998). Drucker (1954) já destacava essa importância, ressaltando ser a inovação uma das funções essenciais para a empresa atingir seu objetivo superior de satisfação dos clientes.

Contudo, a despeito do grande número de publicações específicas sobre os temas de orientação para mercado e performance empresarial, toma-se como considerável o espaço ainda existente para a investigação da mediação que o sucesso de novos produtos pode exercer na relação entre estes construtos (BAKER; SINKULA, 2005).

Neste sentido, este artigo apresenta os detalhes de um estudo que objetivou investigar empiricamente a relação entre orientação para o mercado, sucesso de novos produtos e duas das principais dimensões da performance organizacional, quais sejam, lucratividade e participação de mercado. Os resultados encontrados trazem relevan- tes implicações acadêmicas e gerenciais, contribuindo para a ampliação dos trabalhos que abordam esses temas como modelos integrados.

\section{Referencial teórico}

A linha teórica seguida pelo presente estudo foi aquela iniciada por Sinkula (1994). Em seu estudo inicial sobre o tema, Sinkula (1994) apresentou uma série de proposições a respeito da relação entre processamento de informações de mercado e performance. Ainda que sem mencionar explicitamente o tema de orientação para mercado, Sinkula (1994) foi um dos primeiros autores que buscou relacionar os construtos de orientação para mercado, aprendizagem organizacional e performance. A essência das proposições de Sinkula (1994) foi a de que o conhecimento a respeito do processamento de informações de mercado pode ser substancialmente aprimorado se considerados os princípios definidos pela aprendizagem organizacional.

Sinkula, Baker e Noordewier (1997), dando continuidade ao trabalho de Sinkula (1994), realizaram um estudo com o objetivo de mapear e testar as relações entre a orientação para aprendizagem, os processos de geração e disseminação de informações de mercado e o dinamismo do programa de ações organizacionais, ou seja, o grau com que a organização efetua mudanças nas suas estratégias de mercado. Os resultados do estudo registraram influência positiva da intensidade dos processos de geração e disseminação de informações de mercado sobre o dinamismo do programa de ações organizacionais.

Em trabalho posterior, Baker e Sinkula (1999a) estenderam o seu esquema teórico inicial e propuseram um novo modelo que explicitamente relacionou os construtos de orientação para aprendizagem, orientação para mercado e performance organizacional. Sinteticamente, Baker e Sinkula (1999a) aglutinaram os construtos de geração e disseminação de informações de mercado em um único construto de orientação para mercado. Complementarmente, ampliaram o construto anterior de dinamismo do programa de ações organizacionais para um construto de performance organizacional. A conclusão da pesquisa de Baker e Sinkula (1999a) foi a de que a orientação para aprendizagem afeta indiretamente a performance organizacional, na medida em que incrementa a qualidade da postura de orientação para mercado da organização e influencia diretamente a performance organizacional, uma vez que ela facilita a aprendizagem de ciclo duplo, promovendo a inovação de produtos, procedimentos e sistemas.

Como seqüência de pesquisa, Baker e Sinkula (1999b), declaradamente influenciados pelos trabalhos de Han, Kim e Srivastava (1998) e Hurley e Hult (1998), inseriram o 
construto de sucesso de novos produtos no modelo testado pelo seu estudo anterior. As hipóteses configuradas no novo modelo teórico foram testadas e comprovadas, denotando que "a orientação para mercado e a orientação para aprendizagem são a chave para o sucesso da performance dirigida pela inovação" (BAKER; SINKULA, 1999b, p.14). Essencialmente, as hipóteses postulavam o efeito mediador do sucesso de novos produtos na relação entre a orientação para o mercado e a performance empresarial, além do efeito direto da orientação para aprendizagem sobre os três construtos anteriores. A conclusão mais controversa, no entanto, foi a de inexistência de efeito direto da orientação para mercado sobre a performance organizacional, diferentemente dos resultados de diversas pesquisas anteriores. Baker e Sinkula (1999b), no entanto, ressaltaram que os resultados de seu estudo deveriam ser mais amplamente verificados e, caso replicados, sugeriram que novas pesquisas devem ser realizadas para o aprofundamento das razões de tais resultados.

Baker e Sinkula (2005), na seqüência de sua pesquisa, apontam algumas possíveis explicações para o referido resultado controverso de seu estudo anterior. Postulam esses autores que, na maioria das pesquisas empíricas, a orientação para mercado tem sido relacionada com medidas de performance independentes, com pouco foco em esquemas conceituais que incluam orientação para mercado e múltiplas medidas de performance. As exceções para esta tendência são estudos que demonstraram o efeito mediador de dimensões intermediárias de performance na relação da orientação para mercado com medidas finais (ou terminais) de performance (PELHAM, 1997; HOMBURG; PFLESSER, 2000), bem como alguns estudos que identificaram um papel mediador do sucesso de novos produtos na relação entre orientação para mercado e performance (ATUAHENE-GIMA, 1995; BAKER; SINKULA, 1999b; HAN, KIM; SRIVASTAVA, 1998).

Assim, a principal argumentação de Baker e Sinkula (2005) quanto aos resultados inesperados de seu estudo anterior (BAKER; SINKULA, 1999b) é a de que as medidas agregadas de performance final afetam o entendimento da natureza específica dos efeitos mediadores de variáveis como o sucesso de novos produtos na relação entre a orientação para o mercado e a performance. Nesta linha, a partir de extensa revisão da literatura nos principais journals internacionais da área, Baker e Sinkula (2005) concluíram que 94\% dos estudos empíricos que examinaram o efeito da orientação para o mercado no sucesso de novos produtos relataram uma relação positiva e significativa. Por outro lado, apenas $68 \%$ dos estudos que investigaram a relação entre orientação para o mercado e lucratividade relataram resultados positivos e tão somente $33 \%$ dos trabalhos que avaliaram a relação entre orientação para mercado e participação de mercado relataram resultados positivos.

Os estudos de Baker e Sinkula (1999b) e de Han, Kim e Srivastava (1998) apontam para o sucesso de novos produtos como um elemento-chave do sucesso sustentável e da performance empresarial, decorrendo em um aumento de interesse de estudos relacionados a explorar os antecedentes da inovação (HURLEY E HULT, 1998), assim como barreiras e facilitadores ao processo de inovação (HENARD E SZYMANSKI, 2001).

Desta forma, Baker e Sinkula (2005) propõem um novo modelo teórico (Figura 1), contemplando dimensões da performance organizacional e suas relações com a orientação para mercado e sucesso de novos produtos, dentro de uma visão de rede nomológica. Na concepção teórica de Baker e Sinkula (2005), consistente com outras formulações recentes (ATUAHENE-GIMA, 1996; HAN et al. 1998) e sustentada em seus trabalhos anteriores (BAKER; SINKULA, 1999a, 1999b), o sucesso de novos produtos é diretamente influenciado pelo grau de orientação para o mercado da organização. Empresas orientadas ao mercado são mais propensas a desenvolver novos produtos que ofereçam benefícios únicos ao consumidor, além de apresentarem qualidade superior em relação aos produtos competidores (JAWORSKI; KOHLI, 1993; PELHAM, 1997). Assim a primeira hipótese do

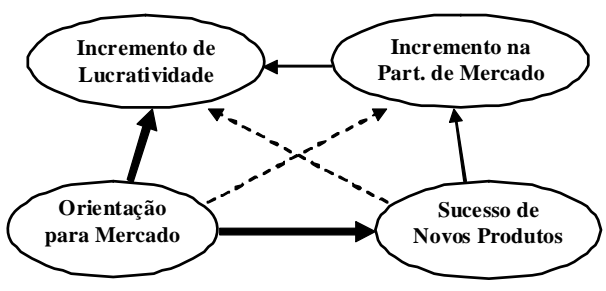

modelo é:

H1: Há uma relação positiva entre orientação para o mercado e o sucesso de novos produtos.

Figura 1 - Modelo Teórico Fonte: Baker e Sinkula (2005)

Por outro lado, apesar de diversos estudos sustentarem que todos os efeitos da orientação para o mercado sobre a performance financeira das organizações são mediados pelo sucesso de novos produtos, Baker e Sinkula (2005) argumentam que deve haver um efeito direto da orientação para o mercado sobre a performance financeira, na medida em que os benefícios de uma forte orientação para mercado afetam positivamente as decisões dos compostos de marketing em desenvolvimento, envolvendo a seleção do mercado alvo, as estratégias de precificação, de distribuição e promoção. Esta sinergia reflete uma competência básica das empresas orientadas ao mercado, qual 
seja, a de agregar valor através da diferenciação e direcionamento dos produtos a segmentos ou nichos específicos, permitindo a prática de preços e margens mais elevadas. Sendo assim, a segunda hipótese proposta no modelo é:

H2: Há uma relação positiva entre orientação para o mercado e lucratividade.

No entanto, embora Hunt e Morgan (1995) afirmem que a orientação para o mercado pode ser uma fonte para a vantagem competitiva, Prahalad e Hamel (1994) e Day (1994) postulam que a vantagem competitiva sustentável resulta de uma consolidação de recursos e capacidades múltiplas. Segundo Baker e Sinkula (2005), esta é, ao menos parcialmente, uma das razões pelas quais apenas um terço dos estudos empíricos pesquisados relata uma relação positiva entre a orientação para o mercado e a participação de mercado. Para Baker e Sinkula (2005), uma relação direta entre orientação para o mercado e participação de mercado se apresenta como tênue porque combina apenas um recurso com uma fonte de vantagem competitiva. Uma relação indireta entre a orientação para mercado e a participação de mercado, mediada pelo sucesso de novos produtos, parece ser mais robusta, na medida em que representa uma aglutinação da orientação para o mercado com outras capacidades relacionadas ao Marketing, expressas propriamente pelo sucesso da inovação (BAKER; SINKULA, 2005). Neste sentido, a terceira hipótese proposta é:

H3: O efeito da orientação para o mercado sobre a participação de mercado é mediado pelo sucesso de novos produtos.

Por outro lado, a habilidade de incrementar a participação de mercado é descrita por Venkatraman e Prescott (1990) como sendo a principal influenciadora da lucratividade. Segundo Baker e Sinkula (2005), há evidências indiretas de que os novos produtos mais lucrativos são aqueles que aumentam a participação de mercado, apesar da ausência de demonstrações empíricas em pesquisas anteriores. Assim, a quarta hipótese sugerida por Baker e Sinkula (2005) se constitui em uma extensão da base teórica, qual seja:

H4: O efeito do sucesso de novos produtos sobre a lucratividade é mediado pela participação de mercado.

Por fim, evidencia-se a necessidade de validação de tal modelo no contexto brasileiro a partir de constatações das diferenças culturais entre o perfil do executivo brasileiro e do norte-americano, baseadas na análise da sua postura individual de tomada de decisão (ZANELA, FREITAS; BECKER, 1998) ou na própria formação histórica da cultura organizacional de cada país (LOPES, 1998). Os procedimentos metodológicos seguidos para a verificação de tal modelo teórico são descritos a seguir.

\section{Método}

O método aplicado na realização desta pesquisa, dada a sua natureza descritiva, foi o levantamento do tipo survey cross-sectional, seguindo recomendações de Churchill (1999) e Malhotra (2001). A técnica utilizada para a avaliação do modelo proposto foi a análise fatorial confirmatória, através do uso de modelagem de equações estruturais (HOYLE, 1995; HAIR et al., 1998).

Para Hair et al. (1998), a técnica de modelagem de equações estruturais é particularmente útil para verificar modelos teóricos mais complexos, nos quais variáveis dependentes se tornam independentes em relações de dependência subseqüentes. Essencialmente, a modelagem de equações estruturais é uma abordagem estatística abrangente que permite o teste de hipóteses sobre as relações entre variáveis observáveis e latentes (HOYLE, 1995).

Os detalhes quanto à elaboração dos instrumentos de coleta aplicados, bem como quanto ao processo de coleta de dados e às análises realizadas, são apresentados a seguir.

\subsection{Elaboração do instrumento de coleta}

A validação do modelo teórico proposto exigiu a elaboração de instrumento de coleta para a mensuração dos seguintes construtos: orientação para o mercado; sucesso de novos produtos; lucratividade; e participação de mercado.

Para operacionalização do construto de orientação para o mercado foi utilizada a escala de Narver e Slater (1990) por apresentar propriedades similares com as escalas de Kohli, Jaworski e Kumar (1993) e de Deshpandé, Farley e Webster (1993) (DESHPANDÉ; FARLEY, 1998), sendo composta por um conjunto total de quinze indicadores, respeitando-se a sua configuração original, através da aplicação de escala do tipo Likert de 5 pontos (1-discordo totalmente a 5- concordo totalmente).

Para mensurar o grau de sucesso de novos produtos, participação de mercado e lucratividade, foram adotadas as operacionalizações sugeridas por Baker e Sinkula (2005). Segundo esses autores, o construto de sucesso de novos produtos foi formado por três variáveis relacionadas ao pioneirismo no mercado, à taxa de introdução de novos produtos e ao grau de sucesso dos novos produtos em relação ao principal concorrente. A participação de mercado foi composta dos indicadores de participação de mercado e de crescimento nas vendas em relação ao principal concorrente. Já a lucratividade foi mensurada por três itens - mudança nos níveis gerais de lucro em 
relação ao último período fiscal, mudança nas margens de lucro e mudança no retorno sobre ativos. Todos os indicadores foram medidos com uma escala de 5 pontos.

A validação de conteúdo do instrumento de coleta foi efetuada através do método de juízes, acadêmicos e práticos (Malhotra, 2001). Os juizes foram dois acadêmicos com experiência na aplicação dos conceitos da área de Marketing. Complementarmente, o instrumento de coleta de dados foi pré-testado em um conjunto de empresas da população-alvo, representando diferentes portes e localizações geográficas, com o objetivo de identificar e eliminar problemas potenciais de entendimento e preenchimento.

Por indicação de Hair et al. (1998) e Garver e Mentzer (1999), a avaliação da confiabilidade do instrumento foi confirmada através do cálculo de confiabilidade (acima de 0,70 ) e de variância extraída (acima de 0,50), a partir da soma das cargas das variâncias padronizadas e dos erros de mensuração das variáveis, além do cálculo do Alfa de Cronbach (acima de 0,70).

\subsection{Coleta de dados}

A forma de coleta adotada, dada a extensão territorial envolvida na pesquisa, foi a de questionário via correio. A população alvo do estudo foi o conjunto de empresas brasileiras de setores econômicos industriais e de serviços, com mais de 30 funcionários, respeitando o padrão aplicado por Baker e Sinkula (2005). Foram enviados 3.000 questionários para empresas selecionadas da população alvo, com base em cadastro adquirido junto à Dun \& Bradstreet do Brasil. O retorno de questionários válidos se aproximou de 10\%, constituindo uma amostra de 293 casos válidos.

O destino do questionário foi a alta administração das empresas envolvidas, sendo encaminhado para o executivo de contato de cada empresa cujo nome consta do referido cadastro. Este procedimento partiu da premissa de que há forte correlação entre as informações prestadas por informantes-chave (respondentes únicos) e por respondentes múltiplos (JAWORSKI; KOHLI, 1993; HOMBURG, WORKMAN; KROHMER, 1999).

Dado o método de coleta utilizado, foi realizada uma análise do viés dos não-respondentes através do teste de comparação entre ondas ( $1^{\mathrm{a}}$ onda após o envio do questionário e $2^{\mathrm{a}}$ onda após a pós-notificação) e da comparação entre as características das empresas respondentes e não respondentes (dados relativos ao número de funcionários, localização geográfica e classificação pelo código de atividade). Os resultados obtidos pelos dois procedimentos indicaram a inexistência de viés nos dados colhidos.

\section{Análise dos resultados}

Neste estudo, a avaliação das relações estruturais seguiu os passos recomendados por Hair et al. (1998), utilizandose o software estatístico AMOS ${ }^{e}$. O modelo analisado considerou todos os construtos como sendo de primeira ordem (BAGOZZI e EDWARDS, 1998). Tal simplificação do modelo foi realizada para a obtenção de parcimônia na estimação dos parâmetros, dado o tamanho da amostra e a complexidade do modelo integrado. Foram seguidas, para tanto, as recomendações de Bagozzi e Edwards (1998) para o processo de agregação total. Neste caso, cada construto foi representado pela soma dos valores medidos através de seus indicadores, transformando-se assim de uma variável latente em uma variável observada.

$\mathrm{O}$ exame das propriedades relativas à validade dos construtos, quais sejam, a unidimensionalidade, a confiabilidade, a validade convergente e a validade discriminante, seguiu recomendações de Garver e Mentzer (1999). Todos os construtos envolvidos no estudo demonstraram validade de construto.

Os indicadores de estimação utilizados para a validação do modelo foram selecionados do conjunto proposto por Hair et al. (1998). Os indicadores considerados têm sido freqüentemente empregados em artigos que utilizam modelagem de equações estruturais para avaliar o construto de orientação para o mercado (FRITZ, 1996; PELHAM, 1997; BHUIAN, 1998; BAKER e SINKULA, 1999b). Destaca-se que existem várias medidas de ajustamento desenvolvidas, absolutas e comparativas, não existindo, portanto, um único coeficiente que resuma o ajustamento do modelo. Além disso, não existem parâmetros rígidos para essas medidas para aceitação ou rejeição de um modelo, dependendo de fatores como a complexidade do modelo proposto.

As medidas absolutas de ajustamento aplicadas, que determinam o grau em que o modelo geral prediz a matriz de covariância ou de correlação observada (HAIR et al., 1998), foram: Qui-quadrado sobre graus de liberdade $\left(\chi^{2} /\right.$ GL), Índice de Adequação de Ajustamento (GFI) e Erro de Aproximação do Quadrado Médio da Raiz (RMSEA). Já as medidas comparativas de ajustamento, que comparam o modelo proposto ao modelo nulo ( null model), foram: Índice de Adequação de Ajustamento Ajustado (AGFI), Índice de Tucker-Lewis (TLI) e Índice de Ajustamento Comparativo (CFI).

Na aplicação da técnica de modelagem de equações estruturais foram analisadas as medidas de ajustamento do modelo teórico, o nível de significância e a magnitude dos coeficientes de regressão estimados para as relações propostas pelas hipóteses do modelo. Para tanto, seguiu- 
se a recomendação explícita oferecida pela empresa SmallWaters Corp., produtora do software estatístico AMOS $^{e}$, utilizado na pesquisa. Dado o diagnóstico de não normalidade não substancial dos dados da amostra (skewness menor ou igual a dois e kurtosis menor ou igual a sete; HOYLE, 1995) e do uso de variáveis categóricas do tipo Likert com cinco ou mais pontos, a empresa sugere que seja aplicada a abordagem maximum likelihood(ML), considerando a variável categórica como intervalar e aplicando o processo de estimação de bootstrapping para a correção dos erros padronizados.

Conforme postulado por Hoyle (1995) e Hair et al. (1998), a aplicação da análise fatorial confirmatória foi precedida de uma avaliação da correlação entre os construtos do modelo para identificação de possíveis relações não incluídas no modelo, ou ainda, a fragilidade das relações teoricamente propostas. A análise da correlação apontou que existe correlação significativa $(p<0,01)$ entre todos os construtos inseridos no modelo. Estes resultados foram sintetizados através de uma análise de correlação considerando as escalas agregadas (summated scales; HAIR

\begin{tabular}{rcccc}
\hline & Lucratividade & $\begin{array}{c}\text { Participacãao } \\
\text { de Mercado }\end{array}$ & $\begin{array}{c}\text { Sucesso de } \\
\text { Novos Produtos }\end{array}$ & $\begin{array}{c}\text { Orientacão para o } \\
\text { Mercado }\end{array}$ \\
\hline Lucratividade & $\mathbf{0 , 9 3}$ & & & \\
Participação de Mercado & 0,344 & $\mathbf{0 , 8 3}$ & & \\
Sucesso de Novos Produtos & 0,251 & 0,502 & $\mathbf{0 , 8 1}$ & $\mathbf{0 , 8 8}$ \\
Orientação para o Mercado & 0,184 & 0,135 & 0,195 & \\
\hline
\end{tabular}

et al., 1998), escalas estas representativas dos construtos em análise. A Tabela 1 apresenta os coeficientes de correlação registrados, além dos coeficientes de Alfa de Cronbach para cada um dos construtos analisados.

Tabela 1 - Correlação entre os Construtos Fonte: Coleta de dados

Nota: todas as correlações foram significativas ao nivel de 0,01 . Os números na linha diagonal representam o coeficiente alpha calculado para cada um dos construtos.

O cálculo dos índices de ajustamento demonstrou um bom ajustamento do modelo teórico, de acordo com o exame das medidas de ajustamento. O teste de Qui-Quadrado, na sua relação com os graus de liberdade, manifestou valor aceitável $(1,791)$. O RMSEA esteve dentro dos padrões de ajustamento $(0,052)$. As demais medidas (GFI =0,983; $\mathrm{AGFI}=0,953$; $\mathrm{TLI}=0,985$; e $\mathrm{CFI}=0.993$ ) foram todas superiores a 0,9, demonstrando ajustamento. Estes resultados registraram a adequação do modelo teórico para a amostra considerada segundo Hair et al. (1998).

O exame da significância e magnitude dos parâmetros estimados (cargas fatoriais padronizadas) permitiu a verificação das relações estabelecidas no modelo teórico, uma vez confirmado o seu ajustamento. A Tabela 2 relaciona os parâmetros em questão. Em termos gerais, a identificação da significância de um coeficiente de regressão (parâmetro estimado) é realizada pela análise do seu respectivo $t$-value. Valores de $t$-values superiores a 1,96 definem um nível de significância de no mínimo 0,05 (DUNN, SEAKER e WALLER, 1994; GARVER e MENTZER, 1999). A constatação de um coeficiente de regressão significativo implica a consideração de que a

\begin{tabular}{|c|c|}
\hline Relação & Cargas Fatoriais Padronizadas \\
\hline Orientaç̃o para o Mercado >> Sucesso de Novos Produtos & $0,195(3,404) * *$ \\
\hline Sucesso de Novos Produtos >> Participação de Mercado & $0,538(9,064) * *$ \\
\hline Orientacão para o Mercado $>>$ Participacão de Mercado & $0,038(0,678)$ \\
\hline Orientação para o Mercado >> Lucratividade & $0,133(2,324) *$ \\
\hline Participação de Mercado >> Lucratividade & $0,365(4,620)^{* *}$ \\
\hline Sucesso de Novos Produtos $>>$ Lucratividade & $0,022(0,319)$ \\
\hline
\end{tabular}

relação entre as duas variáveis se comprova empiricamente (HAIR et al., 1998) e, no caso da avaliação positiva ou satisfatória das medidas de ajustamento, destaca a validade preditiva do modelo (GARVER e MENTZER, 1999).

Tabela 2 - Parâmetros Estimados do Modelo Agregado Fonte: coleta de dados

Nota: Os valores apresentados representam a carga fatorial registrada para cada relação e o respectivo $t$-value (entre parênteses).

** $\mathbf{p}<0,01 ; * \mathbf{p}<0,05$

\subsection{Verificação das hipóteses}

As hipóteses $\mathbf{H 1}$ e $\mathbf{H} 2$ que propunham, respectivamente, uma relação positiva entre orientação para o mercado e o sucesso de novos produtos e uma relação positiva entre orientação para o mercado e lucratividade, foram suportadas pelo exame das cargas fatoriais da relação entre os referidos construtos. $\mathrm{O}$ efeito positivo e significativo registrado pela orientação para o mercado sobre o sucesso de novos produtos $(\beta=0,195, p<0,01)$ foi relativamente superior ao registrado pela mesma orientação para o mercado sobre o lucratividade $(\beta=0,133, p<0,05)$. Para Baker e Sinkula (2005), este resultado é consistente com as conclusões dos estudos anteriores, nos quais se verificou que praticamente todas as pesquisas que testaram a relação entre orientação para o mercado e sucesso de novos produtos a confirmaram, porém dos estudos que testaram a relação entre orientação para mercado e lucratividade, somente cerca de $70 \%$ identificaram a sua efetividade.

A hipótese H3, a qual previa um efeito indireto da orientação para o mercado sobre a participação de mercado, com a mediação do sucesso de novos produtos, foi suportada. Note-se que há uma relação positiva entre a orientação para o mercado e o sucesso de novos produtos, conforme registrado na hipótese $\mathrm{H} 1$, um efeito positivo e significativo do sucesso de novos produtos sobre a participação de mercado $(\beta=0,538, p<0,01)$, mas não foi registrada relação significativa entre a orientação para o mercado e a participação de mercado $(\beta=0,038, p=$ 0,498).

Na mesma linha, a hipótese $\mathbf{H 4}$, que previa um efeito indireto do sucesso de novos produtos sobre a lucratividade, com a mediação da participação de mercado, foi suportada. O impacto do sucesso de novos produtos sobre a participação de mercado se mostrou positivo e significativo $(\beta=0,538, p<0,01)$, bem como o efeito direto da 
participação de mercado sobre a lucratividade $(\beta=0,365$, $p<0,01)$, enquanto a relação entre o sucesso de novos produtos e a lucratividade não foi significativa $(\beta=0,022$, $p=0,750)$

Um procedimento alternativo foi aplicado para a verificação da característica de mediação das variáveis envolvidas nas hipóteses $\mathrm{H} 3$ e H4, qual seja, a verificação dos coeficientes de regressão entre a variável dependente e a independente em dois momentos distintos: sem a variável mediadora e com a sua inserção também como variável independente no cálculo da regressão. Segundo Tabachnick e Fidell (1996), quando uma variável $B$ tem mediação pura na relação entre uma variável $A$ e uma variável $C$, o coeficiente de regressão de $A$ em relação a $C$ é significativo sem a presença da variável $B$, deixando de ser significativo quando da inserção da variável $B$ também como variável independente no cálculo da regressão. Assim, em relação à hipótese $\mathrm{H} 3$, quanto ao efeito da orientação para o mercado sobre a participação de mercado, sem a inserção da variável de sucesso de novos produtos, o cálculo do coeficiente de regressão se mostrou significativo $(\beta=0,160, p=0,06)$. Contudo, com a inclusão da variável sucesso de novos produtos (esta com efeito significativo sobre a participação de mercado - $\beta=0,356$, $p<0,01$ ), o coeficiente de regressão da orientação para o mercado sobre a participação de mercado se tornou não significativo $(\beta=0,090, p=0,103)$. Resultados similares foram encontrados na análise da hipótese H4, no que tange ao efeito do sucesso de novos produtos sobre a lucratividade, mediado pela participação de mercado. Sem a inserção da variável de participação de mercado, o cálculo do coeficiente de regressão do sucesso de novos produtos sobre a lucratividade se mostrou significativo $(\beta=0,251, p<0,01)$. Contudo, com a inclusão da variável de participação de mercado (esta com efeito significativo sobre a lucratividade $-\beta=0,603, p<0,01$ ), o coeficiente de regressão do sucesso de novos produtos sobre a lucratividade se tornou não significativo $(\beta=0,026, p=$ 0,600).

As considerações finais quanto a esses resultados são apresentadas na próxima seção.

\section{Discussão dos resultados}

As diversas correntes teóricas que tratam da orientação para o mercado e sua relação com a performance empresarial são unânimes em afirmar que este tema, apesar de bastante consolidado, ainda apresenta diversas facetas a serem desvendadas. Dentre essas facetas, destaca-se uma questão de pesquisa bastante freqüente nos estudos da área que diz respeito à insuficiência da postura de orientação para o mercado em propiciar às organizações uma vantagem competitiva de longo prazo. Uma série de estudos supracitados discute essa questão específica, manifestando a necessidade de uma ênfase da organização no processo de inovação, paralelamente a sua postura de orientação para o mercado, para a sustentação de uma posição distintiva e duradoura no ambiente competitivo. Neste sentido, este trabalho relacionou as proposições e conclusões de uma linha teórica, encabeçada por Baker e Sinkula (2005), relativas à orientação para o mercado, sucesso de novos produtos e performance organizacional, esta última analisada separadamente em duas de suas principais dimensões - participação de mercado e lucratividade.

O modelo teórico, proposto e verificado, apresentou uma influência indireta, forte e positiva da orientação para o mercado sobre a participação de mercado, mediada pelo sucesso de novos produtos, além do efeito também positivo e significativo da orientação para o mercado sobre a lucratividade. Constatou-se, ainda, a característica de mediação da participação de mercado na relação entre o sucesso de novos produtos e a lucratividade. Esses resultados são absolutamente coerentes com aqueles encontrados no estudo empreendido por Baker e Sinkula (2005).

O efeito direto da orientação para o mercado sobe o sucesso de novos produtos pode ser atribuído à prioridade que as empresas dão à geração de inteligência de mercado no seu processo de decisão. Uma forte orientação para o mercado pode afetar diretamente o desenvolvimento de novos produtos por meio de um ajuste fino entre os desejos dos clientes e os benefícios oferecidos pelo produto (BAKER; SINKULA, 2005). Neste contexto, a orientação para mercado é valiosa para a organização, uma vez que estimula o foco no entendimento e satisfação das necessidades dos clientes, não apenas as expressas, mas também as latentes, através de novos produtos e serviços (DAY, 1994; SINKULA, 1994; SLATER; NARVER, 1995; DICKSON, 1996).

A orientação para o mercado constitui-se em um recurso organizacional fundamental que perpassa toda a organização e seus departamentos, afetando as práticas de administração de recursos humanos, operações e, também, os aspectos de inovação na empresa (HOOLEY et al., 2005). Para Hooley et al. (2005), o aumento da orientação para o mercado leva ao desenvolvimento das capacidades de conexão ou relacionamentos com clientes (DAY, 1994) e ao aumento da habilidade no desenvolvimento e lançamento de novos produtos e serviços, entendido como uma capacidade de inovação de mercado (SLATER; NARVER, 1995; HAN; KIM; SRIVASTAVA, 1998).

As capacidades superiores de inovação de mercado, por sua vez, são fundamentos potenciais para a vantagem competitiva sustentável. Elas são complexas e requerem conexões intangíveis entre as funções, traduzem-se em 
habilidades tácitas e aprendizagem organizacional, de tal modo que suas causas de sucesso são difíceis de desenredar, além de se basearem no fato de que uma inovação em uma empresa não é facilmente duplicável em outra (HAN; KIM; SRIVASTAVA, 1998).

Por outro lado, registrou-se que a orientação para o mercado afeta diretamente a lucratividade. De fato, a orientação para o mercado promove a agregação de valor à marca e fortalece o relacionamento com os clientes pela oferta de melhores serviços, gerenciamento mais eficiente da retenção de clientes, mais efetividade do cross-selling e de programas promocionais, fatores estes que levam a margens de lucro mais altas (SLATER; NARVER, 2000). Esta condição leva a organização a melhores resultados, tais como sucesso de novos produtos, retenção de clientes, crescimento superior e, consequentemente, lucratividade (SLATER; NARVER, 1995). Neste raciocínio, é a orientação para mercado, entre outras variáveis de cultura e clima organizacional, que potencializa o processo de aprendizagem organizacional no sentido de obtenção de uma melhor performance organizacional, em suas várias dimensões (SINKULA, 1994; DICKSON, 1996).

Baker e Sinkula (2005) sustentam, ainda, que a orientação para o mercado pode impactar indiretamente na lucratividade se os seus efeitos no sucesso de novos produtos forem efetivamente convertidos em ganhos na sua participação de mercado. Em termos teóricos, a análise de outros modelos propostos na literatura, semelhantes ao modelo de Baker e Sinkula (2005), como por exemplo o modelo apresentado por Slater e Naver (1995), demonstra que a orientação para mercado influencia diretamente a satisfação de clientes e o sucesso de novos produtos, e que estes, por sua vez, influenciam o crescimento de vendas e a lucratividade. Esta configuração teórica foi ampliada no estudo de Hurley e Hult (1998) pela inserção do construto de capacidade de inovação (inovativeness), como uma variável de impacto no sucesso de novos produtos. Indo além, a habilidade no desenvolvimento de novos ou melhores produtos e serviços, por meio do sucesso de novos produtos, culmina em níveis mais efetivos de satisfação e lealdade de clientes (MAVONDO; CHIMHANZI; STEWART, 2005), em melhores resultados de volume de vendas e participação de mercado (MOORE, 1991; HOOLEY et al., 2005), bem como em um impacto positivo na performance financeira (HURLEY; HULT, 1998; BAKER; SINKULA, 2005).

Assim, a partir do diagnóstico encontrado no presente estudo no que tange à relação entre a orientação para o mercado e dimensões da performance empresarial, além das proposições de autores como Baker e Sinkula (2005), Claycomb e Germain (1997), Homburg e Pflesser (2000), Hurley e Hult (1998), Langerak (2003) e Uncles (2000) é possível especular que outras variáveis de cultura e clima organizacional (tais como a capacidade de inovação, ou inovativeness), bem como outras possíveis dimensões intermediárias de performance (tais como a satisfação de clientes), devidamente operacionalizados, podem colaborar para um melhor entendimento ou explicação da rede nomológica concernente ao papel da inovação na relação entre os construtos de orientação para mercado e performance empresarial.

\subsection{Limitações da pesquisa}

Apesar do rigor do método aplicado nessa pesquisa, algumas situações vivenciadas durante o desenvolvimento do trabalho sugerem certas limitações, e a superação de cada uma dessas limitações resultará em recomendações para pesquisas futuras.

"(...) As associações em um modelo de equações estruturais são evidências necessárias, mas não suficientes de relações causais" (HOYLE, 1995, p. 175). Assim sendo, os resultados confirmatórios aqui apresentados e discutidos, concernentes às relações entre os construtos do modelo proposto, devem ser considerados tão-somente como evidências de relação causal entre os referidos construtos.

A percepção de um único respondente por unidade amostral pode distorcer as verdadeiras práticas adotadas na organização. Desta forma, seria importante que estudos futuros considerassem outras possibilidades, tais como respondentes múltiplos, respondentes de outros escalões da organização ou ainda respondentes externos a organização.

Por fim, apesar da preocupação com a análise do viés dos não respondentes, salienta-se que essa pesquisa utilizou amostras não probabilísticas de retorno de correio. Seria interessante que trabalhos futuros utilizassem mais de um método de coleta de dados, observando a aleatoriedade na seleção das unidades amostrais.

\section{Referências}

ATUAHENE-GIMA, Kwaku. An Exploratory Analysis of the Impact of Market Orientation on New Product Performance: A Contingency Approach. Journal of Product Innovation Management, v. 12, n. 4, p. 275-294, 1995.

ATUAHENE-GIMA, Kwaku. Market orientation and innovation. Journal of Business Research, v. 35, n. 2, p. 93-103, Feb. 1996.

BAKER, William E.; SINKULA, James M. Learning Orientation, Market Orientation, and Innovation: 
Integrating and Extending Models of Organizational performance. Journal of Market-Focused Management, v. 4, n. 4, p. 295-308, Dec. 1999 b.

BAKER, William E.; SINKULA, James M. The synergetic effect of Market Orientation and Learning Orientation on Organizational Performance. Journal of the Academy of Marketing Science, v. 27, n. 4, p. 411-27, Fall 1999a.

BAKER, William E.; SINKULA, James M. Market Orientation and the New Product Paradox. The Journal of Product Innovation Management, v. 22, p. 483-502, 2005.

BAKER, William E.; SINKULA, James M. Does Market Orientation Facilitate Balanced Innovation Programs? An Organizational Learning Perspective. The Journal of Product Innovation Management, v. 24, p. 316-334, 2007.

BARCZAK, Gloria; KAHN, Kenneth B., MOSS, Roberta. An Exploratory Investigation of NPD Practices in Nonprofit Organizations. The Journal of Product Innovation Management, v. 23, p. 512-527, 2006.

BHUIAN, Shahid N. Exploring Market Orientation in Banks: An Empirical Examination in Saudi Arabia. Journal of Services Marketing, v. 11, n. 5, p. 317-327, 1997.

CHURCHILL, Gilbert A, Jr. Marketing Research: Methodological Foundations. Orlando: The Dryden Press, 1999.

CLAYCOMB, Cindy; GERMAIN, Richard. Organizational Learning and Performance: an Empirical Test. In: American Marketing Association Winter Educators' Conference, 1997, St. Petersburg (Florida). Proceedings... St. Petersburg: AMA, 1997. p. 94-100.

DAY, George S. The Capabilities of Market-Driven Organizations. Journal of Marketing, v. 58, n. 4, p 37-52, October 1994.

DESHPANDÉ, Rohit; FARLEY, John U. Market-Focused Organizational Transformation in China. Journal of Global Marketing, v. 14, n. 1-2, p. 7-35, 2000.

DESHPANDÉ, Rohit; FARLEY, John U. Measuring Market Orientation: Generalization and Synthesis. Journal of Market Focused Management, v. 2, n. 3, p. 213-232, 1998.

DESHPANDÉ, Rohit; FARLEY, John U.; WEBSTER, Frederick E, Jr. Corporate Culture, Customer Orientation, and Innovativeness in Japanese Firms: A Quadrad Analysis. Journal of Marketing, v. 57, n. 1, p. 23-27, 1993.
DICKSON, Peter R. The static and dynamic mechanics of competition: a comment on Hunt and Morgan's Comparative Advantage Theory. Journal of Marketing, v. 60, n. 3, p. 102-106, 1996.

DRUCKER, Peter F. The Practice of Management. New York: Harper \& Row, 1954.

GARVER, M. S.; MENTZER, J. T. Logistics research methods: employing structural equation modeling to test for construct validity. Journal of Business Logistics, v. 20, n. 1, p. 33-57, 1999.

HAIR, Joseph F., Jr.; ANDERSON, Rolph E.; TATHAM, Ronald L.; BLACK, William C. Multivariate Data Analysis. 5a Ed. New Jersey: Prentice Hall, 1998.

HAN, Jin K.; KIM, Namwoon; SRIVASTAVA, Rajendra $\mathrm{K}$. Market orientation and organizational performance: is innovation a missing link? Journal of Marketing, v. 62, n. 4, p. 30-45, Oct. 1998.

HARMANCIOGLU, Nukhet; McNALLY, Regina C.; CALANTONE, Roger J.; DURMUSOGLU, Serdar S. Your New Product Development (NPD) is Only as Good as Your Process: an Exploratory Analysis of New NPD Process Design and Implementation. R\&D Management, v. 37, n. 5, p. 399-424, 2007.

HOMBURG, Christian; PFLESSER, Christian. A Multiple-Layer Model of Market-Oriented Organizational Culture: Measurement Issues and Performance Outcomes. Journal of Marketing Research, v. 37, n. 4, p. 448-463, 2000.

HOMBURG, Christian; WORKMAN, John P.; KROHMER, Harley. Marketing's influence within the firm. Journal of Marketing, v. 63, n. 2, p. 1-17, Apr. 1999.

HOOLEY, Graham; FAHY, John; GREENLEY, Gordon; BERACS, Jozsef. Market Orientation in the Service Sector of the Transition Economies of Central Europe. European Journal of Marketing, v. 37, n. 1-2, p. 86-107, 2003.

HOOLEY, Graham J.; GREENLEY, Gordon E., CADOGAN, John W.; FAHY, John. The performance impact of marketing resources. Journal of Business Research, v.58, n. 1; pg. 18-36, 2005.

HOYLE, Rick H (editor). Structural Equation Modeling: Concepts, Issues, and Applications. London: SAGE, 1995.

HUNT, Shelby D.; MORGAN, Robert M. The ResourceAdvantage Theory of Competition: dynamics, path dependencies, and evolutionary dimensions. Journal of Marketing, v. 60, n. 2, p. 107-114, 1996. 
HURLEY, Robert F.; HULT, G. Thomas M. Innovation, market orientation, and organizational learning: an integration and empirical examination. Journal of Marketing, v. 62, n. 3, p. 42-54, July 1998.

JAWORSKI, Bernard J.; KOHLI, Ajay K. Market Orientation: Antecedents and Consequences. Journal of Marketing, v. 57, p. 53-71, July 1993.

KAHN, Kenneth B. Market Orientation, Interdepartmental Integration, and Product Development Performance. Journal of Product Innovation Management, v. 18, n. 5, p. 314-323, 2001.

KOHLI, Ajay K.; JAWORSKI, Bernard J. Market Orientation: The Construct, Research Propositions, and Managerial Implications. Journal of Marketing, v. 54, n. 2, p. 1-18, Apr. 1990.

KOHLI, Ajay K.; JAWORSKI, Bernard J.; KUMAR, Ajith. MARKOR: A measure of Market Orientation. Journal of Marketing Research, v. 30, n. 4, p. 467-477, Nov. 1993.

LANGERAK, Fred. An Appraisal of the Predictive Power of Market Orientation. European Management Journal, v. 21, (August), p. 447-464, 2003.

LOPES, Maria A.R.F. A evolução das pesquisas de cultura organizacional à luz da realidade brasileira e moçambicana. In: ENANPAD, 22, 1998, Angra dos Reis (RJ). Anais... Rio de Janeiro: ANPAD, 1998.

MALHOTRA, Naresh K. Pesquisa de Marketing: Uma Orientação Aplicada. Porto Alegre: Bookman, 2001.

NARVER, John C., SLATER, Stanley F.; TIETJE, Brian. Creating a Market Orientation. Journal of MarketFocused Management, v. 2, n. 3, p. 241-255, 1998.

NARVER, John C.; SLATER, Stanley F. The Effect of Market Orientation on Business Profitability. Journal of Marketing, v. 54, n. 4, p. 20-35, Oct. 1990.

PALADINO, Angela. Investigating the Dricers of Innovation and New Product Success: A Comparison of Strategic Orientations. The Journal of Product Innovation Management, v. 24, p. 534-553, 2007.

PATTIKAWA, Lenny H.; VERWAAL, Ernst; COMMANDEUR, Harry R. Understanding New Product Project Performance. European Journal of Marketing, v. 40, n. 11-12, p. 1178-1193, 2006.

PELHAM, Alfred M. Mediating Influences on the relationship between market orientation and profitability in small industrial firms. Journal of Marketing Theory and Practice, v. 5, n. 3, p. 55-76, 1997.

PERIN, Marcelo G.; SAMPAIO, Cláudio H. A Relação entre as Dimensões de Orientação para Mercado e a Performance. In: ENANPAD, 25, 2001, Campinas (SP). Anais... Rio de Janeiro: ANPAD, 2001.

PERIN, Marcelo G.; SAMPAIO, Cláudio H. Orientação para o Mercado, Porte Empresarial e Performance: um estudo longitudinal no setor Eletroeletrônico do Brasil. In: ENANPAD, 27, 2003, Atibaia (SP). Anais... Rio de Janeiro: ANPAD, 2003.

PERIN, Marcelo G.; SAMPAIO, Cláudio H.; FALEIRO, Sandro Nero. ARelação entre Orientação para o Mercado, Orientação para Aprendizagem e Inovação de Produto. In: ENANPAD, 26, 2002, Salvador (BA). Anais... Rio de Janeiro, ANPAD, 2002.

SINKULA, James M. Market Information Processing and Organizational Learning. Journal of Marketing, v. 58, n. 1, p. 35-45, Jan. 1994.

SINKULA, James M; BAKER, William E.; NOORDEWIER, Thomas. A Framework for MarketBased Organizational Learning: Linking Values, Knowledge and Behavior. Journal of the Academy of Marketing Science, v. 25, n. 4, p. 305-318, 1997.

SLATER, Stanley F.; NARVER, John C. Does competitive environment moderate the market orientation performance relationship? Journal of Marketing, v. 58, n. 1, p. 46-55, Jan. 1994.

SLATER, Stanley F.; NARVER, John C. Market Orientation and the Learning Organization. Journal of Marketing, v. 59, n. 3, p. 63-74, July 1995.

SLATER, Stanley F.; NARVER, John C. The Positive Effect of a Market Orientation on Business Profitability: A Balanced Replication. Journal of Business Research, v. 48 , n. 1, p. 69-73, 2000.

TABACHNICK, B.; FIDELL, L. S. Using Multivariate Statistics (3 ${ }^{\mathrm{a}}$ ed.). New York: Harper Collins, 1996.

UNCLES, Mark. Market Orientation. Australian Journal of Management, v. 25, n. 2, p. i-ix, September 2000.

URDAN, André T.; OSAKU, Willian A. (2005). Determinantes do Sucesso de Novos Produtos: Um Estudo de Empresas Estrangeiras no Brasil. In: ENANPAD, 28, 2001, Brasília (DF). Anais... Rio de Janeiro: ANPAD, 2005 .

VENKATRAMAN, N.; PRESCOTT, J. E. The Market Share-Profitability Relationship: Testing Temporal Stability Across Business Cycles. Journal of Management, v. 16 n. 12, 783-806, 1990. 